\title{
Primary giant cell malignant fibrous histiocytoma of the lung: a rare case report and literature review
}

\author{
Zhen $\mathrm{Xu}^{1 \#}$, Wei Qu ${ }^{2 \#}$, Yonghua $\mathbf{Y u}^{2}$ \\ ${ }^{1}$ Graduate School, Shandong First Medical University \& Shandong Academy of Medical Sciences, Jinan, China; ${ }^{2}$ Department of Radiation Oncology \\ Ward 2, Shandong Cancer Hospital Affiliated to Shandong University, Shandong Academic of Medical Sciences, Jinan, China \\ \#These authors contributed equally to this work. \\ Correspondence to: Yong-Hua Yu. Department of Radiation Oncology Ward 2, Shandong Cancer Hospital Affiliated to Shandong University, \\ Shandong Academic of Medical Sciences, Jinan 250000, China. Email: sdwufx@163.com.
}

\begin{abstract}
Malignant fibrous histiocytoma (MFH) usually involves the extremities. Among MFH, primary giant cell malignant fibrous histiocytoma of the lung is extremely rare, with nonspecific symptoms. A 45-year-old woman was first diagnosed as sclerosing hemangioma due to long-term persistent cough and blood in sputum. One year later, the condition became worse and hemoptysis occurred. At the second visit, the same mass was found to be larger than the previous one. Lobectomy was performed and the primary giant cell MFH of the lung was confirmed. Renal and sacrum metastasis occurred six months after operation. The efficacy of hypofractionation radiotherapy and immuno targeted follow-up therapy is significant. At present, there is no standard treatment plan. For the patients in the early stage, surgical resection is the main treatment method; but for the patients in the middle and late stage, the treatment is relatively single. So early diagnosis and treatment are particularly important, In this paper, we first report the use of pembrolizumab combined with anlotinib in a patient with advanced giant cell MFH of the lung, with failure of routine treatment. The development of the disease had successfully delayed and satisfactory results were observed. Therefore, it is very necessary to emphasize the choice of treatment plan to improve the prognosis of patients.
\end{abstract}

Keywords: Treatment; malignant fibrous histiocytoma (MFH); undifferentiated pleomorphic sarcoma; lung; case report

Submitted Mar 25, 2020. Accepted for publication Apr 23, 2020.

doi: $10.21037 /$ tcr-20-2297

View this article at: http://dx.doi.org/10.21037/tcr-20-2297

\section{Introduction}

Malignant fibrous histiocytoma (MFH) is the most common malignant mesenchymal tumor in adults (1), with an onset age between 50 and 70 (2). Its predilection sites are the trunk part of extremities and the deep musculature of the retroperitoneum (3). Although the lung is the most common site of $\mathrm{MFH}$ metastasis (75\%), MFH originating in the lung is extremely rare (4). On account of its complex and heterogeneous pathological characteristics, lack of specific immunohistochemical markers and no lineage specificity, it is difficult for pathologists to agree on a standard for MFH tumor evaluation (5). Therefore, the diagnosis of $\mathrm{MFH}$ is controversial, and the actual incidence is probably between $20 \%$ and $70 \%$ but unknown. The prognosis of the disease is unfavorable, with a high rate of local recurrence rate and distant metastasis. At present, surgery is still the predominant treatment. In this article we report a rare case of primary MFH of the lung with renal, and sacrum metastases, and highlights the challenges in pathological diagnoses and treatment planning. The primary giant cell MFH of the lungs is very rare, which is characterized by high invasion, atypical clinical symptoms and imaging findings, so early diagnosis and treatment are very important. Its diagnosis mainly depends on pathology, as well as fine needle aspiration biopsy. In term of the lack of typical pathological manifestations and immunoistochemical 

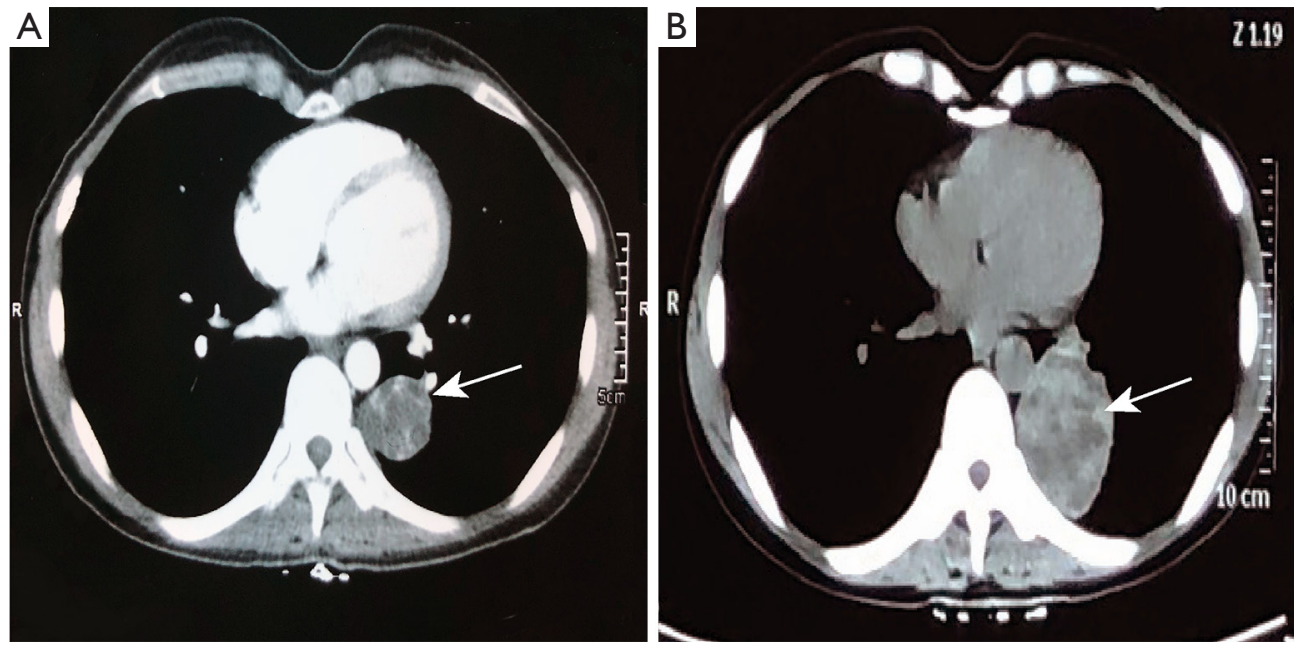

Figure 1 Chest CT of a 45 year old Asian women. (A) The initial CT scan showed a mass with uneven density in the lower lobe of the left lung, $2 \mathrm{~cm} \times 1.8 \mathrm{~cm}$ in size. (B) The same mass half a year later, $7 \times 6 \times 5 \mathrm{~cm}$ in size. CT, computed tomography.

markers, pathological diagnosis is often exclusive. The prognosis of the disease is poor. At present, the main treatment is surgery, and combined therapy may be a better option in the treatment plan. We first used pembrolizumab combined with anlotinib in a patient with advance giant cell MFH of the lung, and the treatment was effective. The findings need to be supported by further clinical studies. We present the following article in accordance with the CARE reporting checklist (available at http://dx.doi.org/10.21037/ tcr-20-2297).

\section{Case presentation}

All procedures performed in studies involving human participants were in accordance with the ethical standards of the institutional and/or national research committee(s) and with the Helsinki Declaration (as revised in 2013). Written informed consent was obtained from the patient.

In March 2018, a 45 years old Asian woman with persistent cough and blood in sputum was admitted to our hospital. Chest computed tomography (CT) scan examination revealed a space-occupying lesion in the lower lobe of left lung, $2 \times 1.8 \mathrm{~cm}$ in size. And contrast enhanced CT showed heterogeneous density of the lesion. The patient was previously healthy, no history of respiratory disease, no habits of smoking and alcohol, and no family history of cancer. She was first misdiagnosed as sclerosing hemangioma considering the clear margin of the mass, without hilar and mediastinal lymph nodes swollen. Six months later, the patient developed symptoms of hemoptysis. CT examination revealed a larger mass, $7 \times 6 \times 5 \mathrm{~cm}$ in size (Figure 1). In order to solve the hemoptysis problem, a lobectomy of left lower lung was performed. No postoperative metastases were observed using the positron emission tomography (PET). And postoperative pathology confirmed the disease as a malignant soft tissue tumor, but the possibility of the malignant giant cell tumor of soft tissue could not be excluded. Another study center considered it as mesenchymal tumor with osteoclastic-like giant cells, similar to giant cell tumor of soft tissue (GCTST) or giant cell MFH. As the patient was not re-examined regularly after surgery, she was admitted to the hospital 7 months later and complained of a continuous backache for 1 month. Thoraco-abdominal CT demonstrated an oval mass with uneven density on the right kidney, $6.8 \times 4.5 \mathrm{~cm}$ in size; another oval lump was also found on the right kidney, without enhancement. Then emission CT (ECT) showed an abnormal uptake of skeletal imaging agent in both left 5th and 6th costae and pelvic soft tissue near the sacrococcygeal region. PET revealed no recurrence in the surgical area, malignant lesions in the right kidney (SUV max 6.6) and in the 3rd and 4th sacral vertebrae (SUV max 5.8), and also in the left transverse process of the 4th lumbar vertebra (SUV max 3.6) (Figure 2). Laboratory data including complete blood count and liver and kidney function revealed no other abnormalities. The biopsy of sacrum mass was lately preformed, which was considered to be sarcoma. However, it was diagnosed as giant cell tumor 

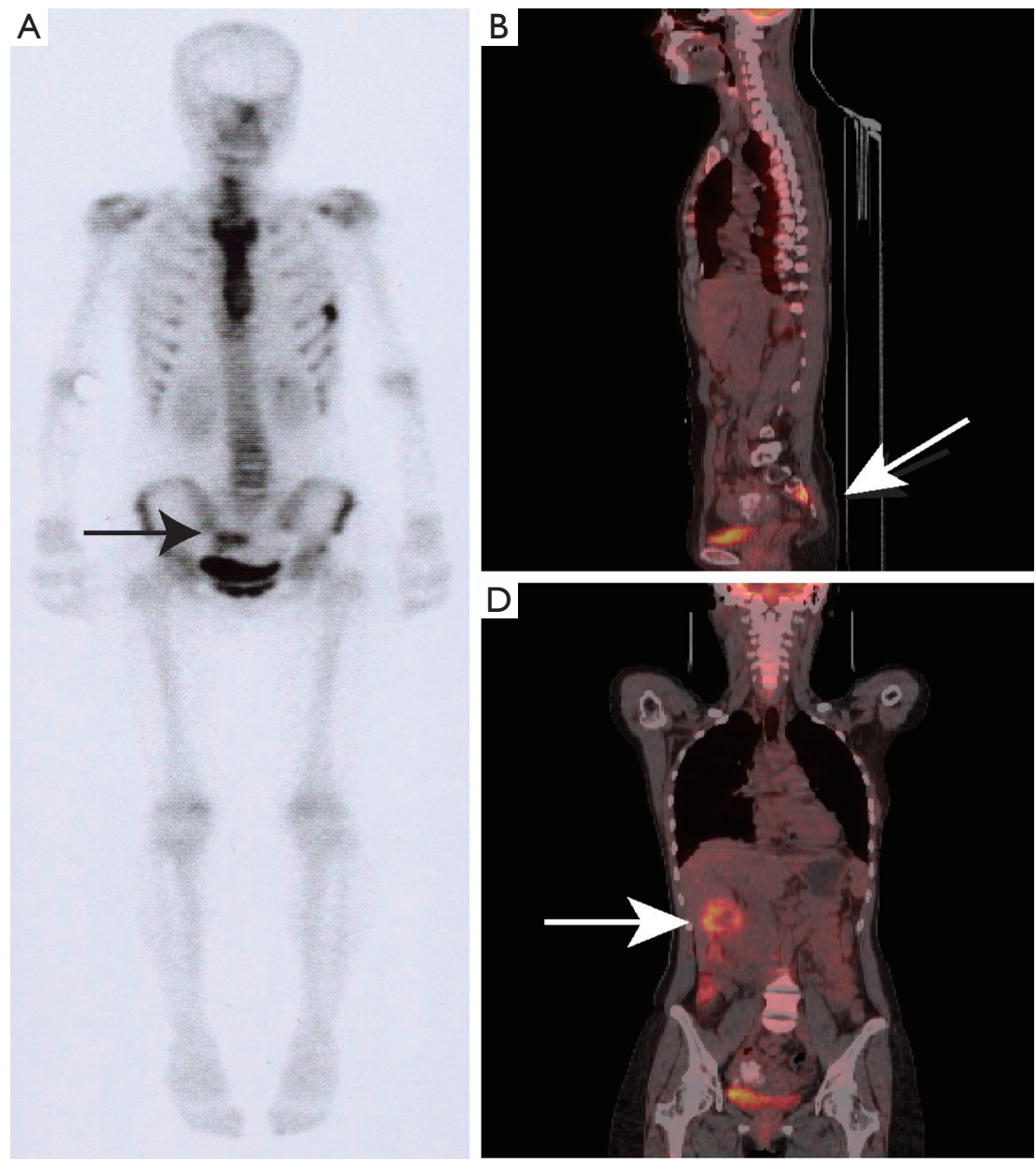

Figure 2 Thoraco-abdominal CT of a 45 years old Asian women 7 months later. (A) ECT imaging shows the abnormal uptake of skeletal imaging agent in pelvic soft tissue near the sacrococcygeal region; (B) PET imaging shows a malignant mass on the 3rd and 4th sacral vertebrae (SUV max 5.8); (C) The metastasis lesion on the right kidney (SUV max 6.6). CT, computed tomography; ECT, emission computed tomography; PET, polyethylene terephthalate; SUV, standardized uptake value.

of bone by pathology consultation. Finally, combined with the clinical features and immunohistochemical analysis, the diagnosis of giant cell MFH was made. The patient was in stage T2aN0M0 (AJCC 8th edition). A total dose of 50 Gy was divided into 10 fraction, and then was delivered into the sacrum mass irradiated locally. During radiotherapy, the patient developed grade II gastrointestinal adverse reactions, manifested as hematochezia and diarrhea. After radiotherapy, CT showed that the right renal mass was larger than that before, about $9.41 \times 6.66 \mathrm{~cm}$ in size. Anlotinib was administered at a dose of $8 \mathrm{mg} /$ day for two weeks followed by 1 -week off-treatment, which was as a cycle. After 2 cycles, the disease progression was reviewed. Bevacizumab combined with epirubicin/ ifosfamide was administered orally once a day, $300 \mathrm{mg}$ every 21 days; the patient received epirubicin at $60 \mathrm{mg} / \mathrm{m}^{2}$ on days 2 and 3 and intravenous ifosfamide at $1,800 \mathrm{mg} / \mathrm{m}^{2}$ from days 1 to 5 of a 21-day cycle. Because the patients had grade II gastrointestinal reactions during the first cycle of chemotherapy, manifested as nausea and vomiting, the treatment in the second cycle was adjusted in epirubicin $100 \mathrm{mg} / \mathrm{m}^{2}$ on days 2 and 3 , intravenous ifosfamide at $1,800 \mathrm{mg} / \mathrm{m}^{2}$ from days 1 to 4 . After 2 cycles, the efficacy of PD (recist 1.1) in the treatment because of liver metastasis was evaluated. Immunotherapy combined with anlotinib was then used. The patient developed Grade II myelosuppression after the first cycle treatment. After 3 months reexamination, it was found that the right renal 

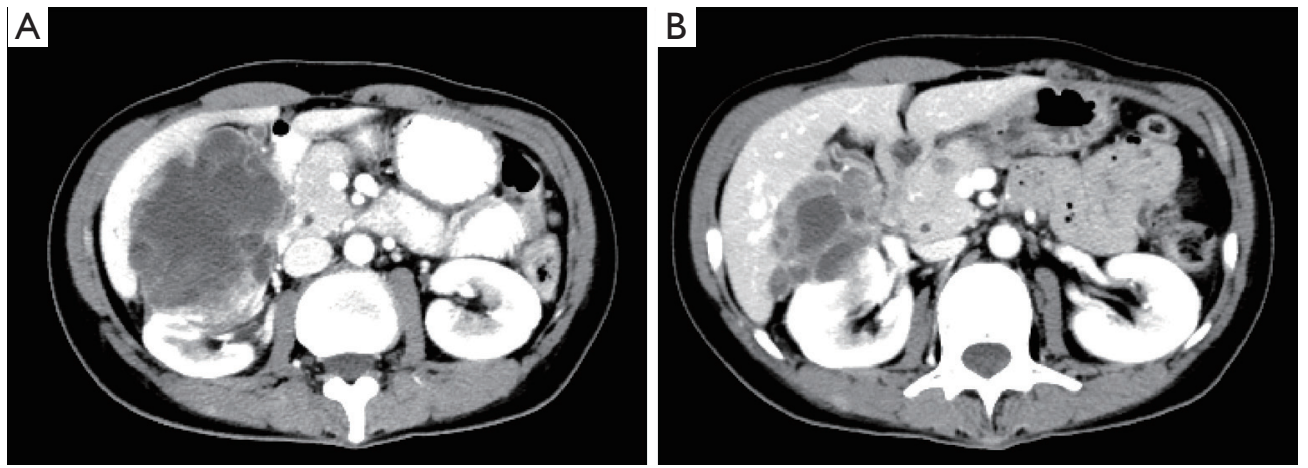

Figure 3 CT of a 45 years old Asian women after radiotherapy. (A) The primary right kidney mass before therapy, 9.41×6.66 in size; (B) after 3 cycles of immunotherapy and targeted treatment, the index of metastatic lesion in the right kidney decreased, $5.02 \times 3.17$ in size. CT, computed tomography.

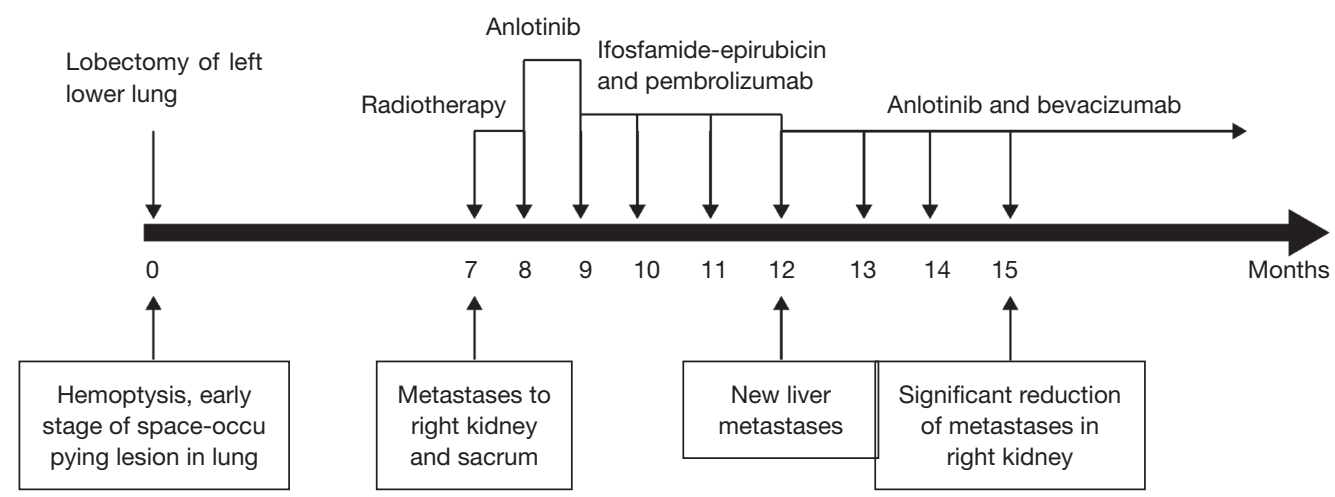

Figure 4 Timeline of treatment process. This figure presents the changes of drug application and the patient's condition.

space was reduced to $5.02 \mathrm{~cm} \times 3.17 \mathrm{~cm}$ (Figure 3). Given that the treatment was effective, the patient returned to the local area for treatment. Follow up was performed every 2 months after discharge, including routine physical examination, contrast enhanced CT of the chest and abdomen, and serum tumor markers. By the end of our paper, follow-up found that the patient was still alive, and the imaging evidence shows that the patient's condition is stable and no new lesions (Figure 4).

\section{Histological findings}

Microscopically, multiple multinucleated osteoclast-like giant cells were evenly distributed on the mononucleated stromal cells. Giant cells contained numerous nuclei with eosinophilic cytoplasm. The pleomorphic stromal cells were spindle-shaped and atypical round, which were similar to the high-grade sarcoma. Nucleoli could be seen in several stromal cells. A small number of stromal cells showed a high degree of mitosis, which is a sign of malignancy. Histological examination suggested diagnosis of giant cell MFH (Figure 5). Immunohistochemical staining for the fine-needle biopsy showed the weakly positive differentiation of sacrum lesions cluster CD68(+), CD10(+), $\mathrm{LCA}(+)$ and $\mathrm{SMA}(+), \mathrm{Ki}-67(+), \mathrm{S} 100$ in the occasional tumor cells, CKpan-CAM5.2-, TTF-1-, CK7-, CK5/6-, $\mathrm{P} 63$ - under the clinical diagnosis of giant cell $\mathrm{MFH}$.

\section{Review of the 3 cases of pulmonary giant cell MFH or UPS in the literature}

PubMed was searched in English for relevant medical literature, with "malignant fibrous histiocytoma" "MFH" "undifferentiated pleomorphic sarcoma" "UPS" "lung" "pulmonary" "giant cell" "osteoclast-like giant cell" and "primary" as keywords. Only three cases found in the 

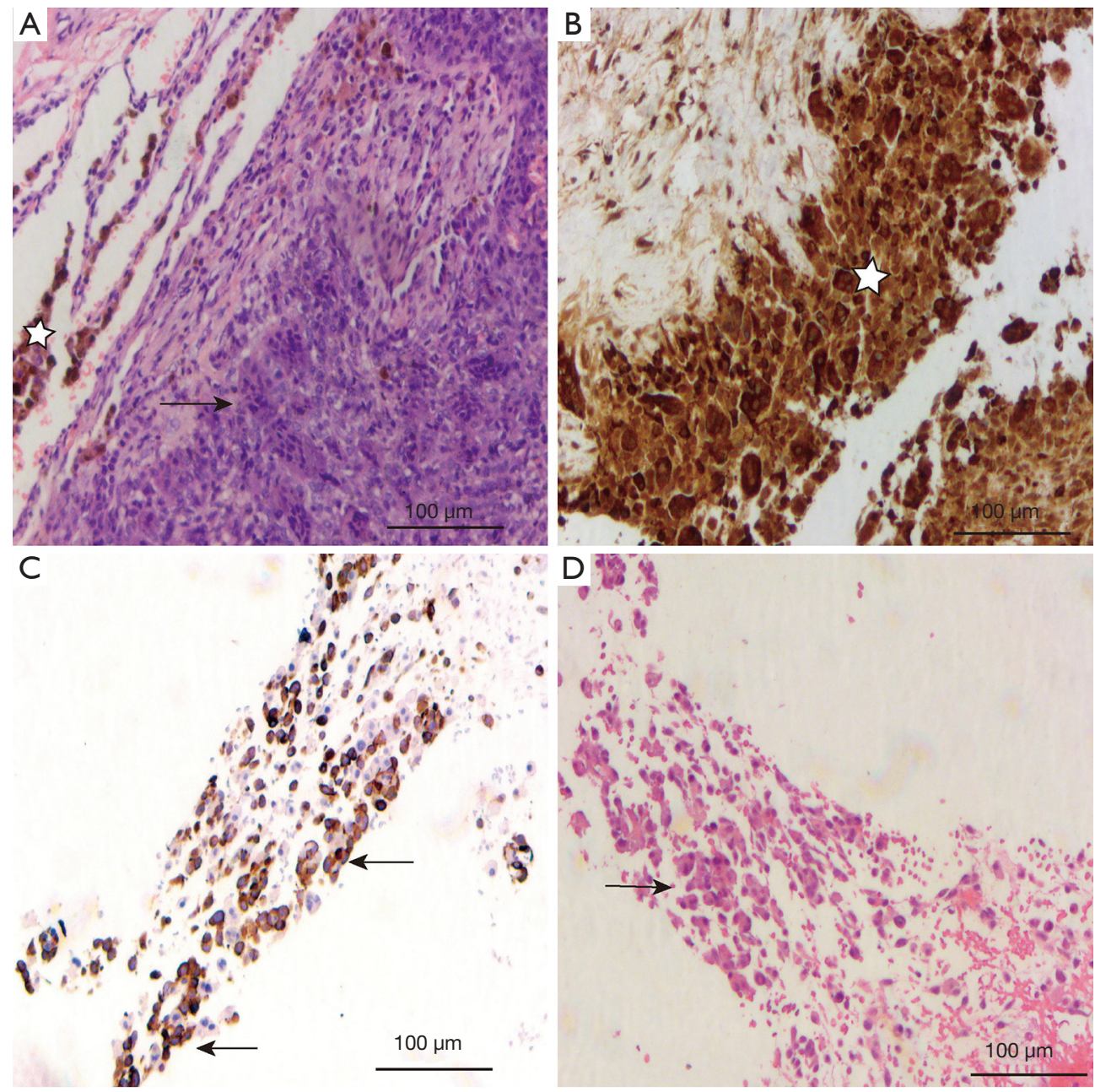

Figure 5 HE chromatogram $(100 \mu \mathrm{m})$. (A) Typical appearance of abundant multinucleated giant cells accompany with mononucleated stromal cells. The brown coarsely granular material of macrophages in the alveolus indicates chronic inflammation (asterisk indicates hemosiderin cells) $(\mathrm{HE} \times 100)$; (B) high CD68 expression of lung mass (asterisk indicates that the tumor cells are CD68 positive) (IHC $\times 100$ ); (C) the sacral mass is positive for SMA, indicating a high-grade sarcomatous transformation (arrow) (IHC $\times 100)$; (D) MFS of sacral metastasis. View of highly atypical cytologic features, shows pleomorphism character $(\mathrm{HE} \times 100)$. HE, hematoxylin-eosin staining; IHC, immunohistochemistry; SMA, smooth muscle actin.

literature met our defined criteria. The clinical features and outcomes are summarized in Table 1.

\section{Discussion}

The conception of MFH was first described by O'Brien and Stout in 1949. These highly invasive soft tissue tumor are characterized by storiform or cartwheel-like growth pattern (6). Pathologists usually classify these tumor into five histologic subtypes: storiform, pleomorphic, giant cell, inflammatory, and myxoid. With the improvement of immunohistochemistry and karyotype analysis as well as the understanding of the disease, the traditional definition of MFH has experienced daunting challenge. It has been proved to be a collection of poorly differentiated tumors without a consistent fibroblast spectrum, and only a subset of them can be reclassified by immunohistochemistry and genetics. Considering the saturation, WHO renamed $\mathrm{MFH}$ as undifferentiated pleomorphic sarcoma (UPS). $\mathrm{MFH}$ accounts for $5 \%$ of adult soft-tissue tumors, as one of the most common tumors in adults. UPS predominantly affects extremities and trunk. The primary lesions in the 
Table 1 Reported cases of giant cell MFH of the lung

\begin{tabular}{|c|c|c|c|c|c|c|c|}
\hline Authors & $\begin{array}{l}\text { Age at } \\
\text { diagnosis } \\
\text { (years) }\end{array}$ & Presentation & $\begin{array}{l}\text { Variant } \\
\text { associated } \\
\text { lesion (s) }\end{array}$ & Size $(\mathrm{cm})$ & Imaging performance & Treatment & Outcome \\
\hline $\begin{array}{l}\text { Kimizuka } \\
\text { G (6) }\end{array}$ & 46 & Cough & Left upper lung & $6 \mathrm{~cm} \times 6 \mathrm{~cm} \times 6 \mathrm{~cm}$ & $\begin{array}{l}\text { X-ray shows a shadow on } \\
\text { the left lung }\end{array}$ & $\begin{array}{l}\text { Surgical } \\
\text { resection }\end{array}$ & $\begin{array}{l}\text { No evidence of } \\
\text { disease at } \\
2 \text { years }\end{array}$ \\
\hline $\begin{array}{l}\text { Juettner } \\
\text { FM (7) }\end{array}$ & 68 & $\begin{array}{l}\text { Collapse and } \\
\text { unconsciousness }\end{array}$ & Left lower lung & $20 \mathrm{~cm} \times 11.5 \mathrm{~cm} \times 15 \mathrm{~cm}$ & $\begin{array}{l}\text { a large paramediastinal } \\
\text { expansion with atelectasis } \\
\text { of the lower lobe and } \\
\text { pleural effusion }\end{array}$ & $\begin{array}{l}\text { Surgical } \\
\text { resection }\end{array}$ & $\begin{array}{l}\text { Died after } \\
1 \text { year }\end{array}$ \\
\hline
\end{tabular}

lungs are extremely rare (7), accounting for about $0.2 \%$ of all lung malignancies. The age of patients varies from 20 to 80 (average age: 62), with a higher incidence in the elderly. UPS has atypical clinical symptoms, with dyspnea and thoracalgia as the most common features; cough, weight loss, fatigue, hemoptysis can also be seen in other cases. As a result, most patients are diagnosed in the advanced stage at the time of presentation. Its imaging performance is also non-specific. The mass observed in CT is mostly atypical, often large, solid and isolated, usually found in the lower and middle lungs. The density can be uneven, and hemorrhagic necrosis can be seen inside. The imaging of these tumors are similar to that of sclerosing hemangioma; it may be difficult to distinguish them, so fine needle biopsy is necessary (8). PET-CT can be used as a diagnostic for exclusion. The diagnosis of malignant fibroids is often excluded.

Due to the complexity of the cells that make up the UPS, the microscopic performance is often diverse, and the tumor cells are often arranged in a pattern of turbine (9). Tumor cell morphology is atypical—spindle-shaped, round or irregular. Microscopically, the tumor cells are deeply stained, mitosis is active, and pathological mitosis is more common. In the tumor tissue, hemorrhagic necrosis is observed, as well as chronic inflammation and osteoclast infiltration. Hemosiderin infiltration can also be found in some pathological specimens. A large number of multinucleated giant cells are often scattered in the tumor tissue, and most of the giant cells are osteoclasts, and such cells have been shown to be derived from mesenchyme. The role of osteoclastoma-like giant cell (OGC) is not fully understood, which may contribute to immune factors such as chemotaxis (10). However, there is also evidence that macrophages may play an important role in tumor metastasis and are associated with poor prognosis $(11,12)$. Tumour-associated macrophages (TAMs) promote tumour progression and metastasis, and inhibit anti-tumour immune responses. Nina Linde has observed in a mouse model of HER2 breast cancer that metastasis of early tumor cells requires the help of macrophages. Tumor cells show no other identifiable line of differentiation, however, immunohistochemistry can also play a role in exclusive diagnosis. The keratins, S100 protein, CD31, CD34, CD68, CD163, desmin, SMA, CD45, CD30 are nondispersive expression, while myogenin, MYOD1, p63, ERG are negative. In addition, non-tumor cells in tumor tissues, such as multinucleated giant cells with positive CD68, pose great challenges for diagnosis. Pathologically it often needs to be identified with the following diseases: dedifferentiated liposarcoma, other high-grade pleomorphic sarcomas melanoma, lymphoma. In pleomorphic liposarcoma, hallmark pleomorphic adipocytes are often able to visually recognize, while in dedifferentiated liposarcoma, welldifferentiated tumor areas are visible. In our case, CD10, CD68, LCA and SM, Ki-67, and S100 are positive, which is consistent with the literature. It is worth mentioning that in our case, the round or elliptical tumor cells are diffusely distributed in the tumor tissue. These cells are more regular in shape and lack obvious pathological mitotic figures, and a large number of osteoclasts are visible. These features are similar to those of GCT-ST, so it is difficult to differentiate 
the pathology of the two. However, benign GCT-ST do not express S100, and high-grade GCT-ST can be equated with giant cell villous fiber groups, which provide the basis for differentiation.

Currently, there is no standard treatment for UPS. The main treatment for this disease is surgical resection plus postoperative radiotherapy. The surgical margin recommended is $4 \mathrm{~cm}$ away from the tumor. According to Yoshida's study, surgical margin are positively correlated with the local recurrence rate (13). The local recurrence rate at the surgical margin of 4 and $2 \mathrm{~cm}$ is $44 \%, 71 \%$. But the function of chemotherapy is unclear (14); $\mathrm{MFH}$ is not sensitive to chemoradiotherapy (15). A previous study has shown that a 5 -year survival rate is $58 \%$ and a 10 -year survival rate is $38 \%$. The traditional first-line chemotherapy regimen is doxorubicin or ifosfamide. The multicenter, single-agent doxorubicin and phase III clinical trial of doxorubicin-ifosfamide chemotherapy in Europe indicates a higher tumor response and overall survival (OS) (16) (OR 9.90, 95\% HR 0.44, 95\%). Therefore, gemcitabine/docetaxel chemotherapy may be a good option for patients with doxorubicin or ifosfamide failure (17). According to the retrospective study of Jee Hung Kim (17), the MFH/UPS efficiency is $50 \%$. However, these chemotherapy regimens are highly toxic which may lead to treatment failure. Local radiotherapy should also be considered but radiotherapy is often limited by the disease location. Anlotinib, as a new oral multi-target inhibitor, has the effects of anti-proliferative, anti-angiogenic and anti-metastatic. It can block vascular endothelial growth factor receptor (VEGFR-2), fibroblast growth factor receptor (FGFR), platelet-derived growth factor receptor (PDGFR), c-Kit, Ret, Aurora-B, c-FMS, and Discoidin domain receptors 1 (DDR1). Wang G's experiments have confirmed that anlotinib can not only effectively inhibit the growth and metastasis of osteosarcoma, but also increase chemo-sensitivity (18). Combined therapy may be another treatment strategy worth considering. It has been reported that combined therapy can prolong the survival of patients with advanced metastases compared with monotherapy. Meena Bedi retrospectively studied 182 patients with soft tissue sarcoma between 2000 and 2010, and found that patients who received comprehensive treatment had a longer survival period of 18 months than those who received monotherapy. In addition, immunotherapy is also worth expecting (19). In a recently published stage II multicenter SARC028 (NCT02301039) study, pembrolizumab has achieved some efficacy in advanced undifferentiated pleomorphic sarcoma and dedifferentiated liposarcoma. Another study indicated that the use of ferumoxytol nanoparticles induced a pro-inflammatory macrophage response and significantly inhibited tumor growth. Superparamagnetic iron oxide induces the transformation of M1 macrophage into M2 macrophages, thereby inducing apoptosis in cancer cells. These apoptotic cancer cells stimulate $\mathrm{M} 1$ to produce $\mathrm{TNF} \alpha$ and nitric oxide (NO) to continuously kill tumor cells (20).

As a highly invasive malignant tumor, the prognosis of this disease is poor, with a 5 -year OS of $65-70 \%$, a local recurrence rate of $19-31 \%$, and a distant metastasis rate of $50 \%$. The mean survival period of patients without surgery is 11.7 months while 23.2 months for patients with surgery. The disease often metastasizes and recurs 12 to 24 months after diagnosis. The most common sites of metastasis are lung (90\%), bones (8\%), and liver (1\%). The prognosis is not related to the tumor cell abnormality, but to the tumor location, stage, immunosuppression, patient's age, pathological type, and tumor size. Tumor size larger than $2 \mathrm{~cm}$ and deep location, cause poor prognosis in the patients under 50 years. Ki-67 expression may be positively correlated with prognostic factors. Si-Qi Qiu1 performed a short-term follow-up of 7 patients. Among the 4 patients with high Ki-67 expression, 2 patients had local recurrence and distant metastasis with a life span of only 6 to 26 months, and 2 patients with low Ki-67 expression had no recurrence or distant metastasis in 145 months.

In our case, the patient showed non-specific symptoms in the early stage such as cough and hemoptysis, so it was misdiagnosed as sclerosing hemangioma. Due to the progressive enlargement of the mass and hemoptysis, the lobectomy was then performed. After the postoperative pathology, it was misdiagnosed as giant cells tumor. However, renal and sacral metastases were found 7 months after surgery which is consistent with relevant research characteristics of metastases that appears within 9 months after surgery.

During subsequent treatment, we observed that the new model of the combination of immune and targeted therapy benefited patients with advanced giant cell MFS. The disease progression was successfully delayed, with well tolerance. The limitation of this study is that it provides only individualized treatment regimens, but lacks corresponding evidence of efficacy, so further studies are required.

\section{Acknowledgments}

Funding: None. 


\section{Footnote}

Reporting Checklist: The authors have completed the CARE reporting checklist. Available at http://dx.doi.org/10.21037/ tcr-20-2297

Conflicts of Interest: All authors have completed the ICMJE uniform disclosure form (available at http://dx.doi. org/10.21037/tcr-20-2297). The authors have no conflicts of interest to declare.

Ethical Statement: The authors are accountable for all aspects of the work in ensuring that questions related to the accuracy or integrity of any part of the work are appropriately investigated and resolved. All procedures performed in studies involving human participants were in accordance with the ethical standards of the institutional and/or national research committee(s) and with the Helsinki Declaration (as revised in 2013). Written informed consent was obtained from the patient.

Open Access Statement: This is an Open Access article distributed in accordance with the Creative Commons Attribution-NonCommercial-NoDerivs 4.0 International License (CC BY-NC-ND 4.0), which permits the noncommercial replication and distribution of the article with the strict proviso that no changes or edits are made and the original work is properly cited (including links to both the formal publication through the relevant DOI and the license). See: https://creativecommons.org/licenses/by-nc-nd/4.0/.

\section{References}

1. Makepeace AR, Cannon SR. Malignant fibrous histiocytoma: the most common soft-tissue sarcoma. Br J Hosp Med 1988;39:122, 124, 126.

2. Patel DP, Gandhi YS, Sommers KE, et al. Primary pulmonary malignant fibrous histiocytoma. Case Rep Pulmonol 2015;2015:381276.

3. Lindberg MR. Diagnostic Pathology: Soft Tissue Tumors, 2nd Ed. 2015:718-21.

4. Liu Y, Chen G, Wu Y, et al. Combined pulmonary lobectomy for surgical treatment of a malignant fibrous histiocytoma of the chest wall: a case report. Diagn Pathol 2014;9:21.

5. Jo VY, Fletcher CD. WHO classification of soft tissue tumours: an update based on the 2013 (4th) edition. Pathology 2014;46:95-104.
6. Kimizuka G, Okuzawa K, Yarita T. Primary giant cell malignant fibrous histocytoma of the lung: a case report. Pathol Int 1999;49:342-46.

7. Juettner FM, Popper H, Sommersgutter K, et al. Malignant fibrous histiocytoma of the lung: prognosis and therapy of a rare disease. Report of two cases and review of the literature. Thorac Cardiovasc Surg 1987;35:226-31.

8. Zanganeh S, Hutter G, Spitler R, et al. Iron oxide nanoparticles inhibit tumour growth by inducing proinflammatory macrophage polarization in tumour tissues. Nat Nanotechnol 2016;11:986-94.

9. Hoda SA, Cheng E. Robbins Basic Pathology. Am J Clin Pathol 2017. doi: 10.1093/ajcp/aqx095.

10. Kong L, Peng W, Liu J, et al. Squamous cell carcinoma of lung associated with osteoclast-like giant cells: report of a case. Int J Clin Exp Pathol 2015;8:11823-25.

11. Winchester D, Lehman J, Tello T, et al. Undifferentiated pleomorphic sarcoma: Factors predictive of adverse outcomes. J Am Acad Dermatol 2018;79:853-59.

12. Kulkarni A, Chandrasekar V, Natarajan SK, et al. A designer self-assembled supramolecule amplifies macrophage immune responses against aggressive cancer. Nat Biomed Eng 2018;2:589-99.

13. Yoshida N, Miyanari N, Yamamoto Y, et al. Successful treatment of malignant fibrous histiocytoma originating in the chest wall: report of a case. Surg Today 2006;36:714-21.

14. Kasper B, Ouali M, van Glabbeke M, et al. Prognostic factors in adolescents and young adults (AYA) with high risk soft tissue sarcoma (STS) treated by adjuvant chemotherapy: a study based on pooled European Organisation for Research and Treatment of Cancer (EORTC) clinical trials 62771 and 62931. Eur J Cancer 2013;49:449-56.

15. Qiu SQ, Wei XL, Huang WH, et al. Diagnostic and therapeutic strategy and the most efficient prognostic factors of breast malignant fibrous histiocytoma. Sci Rep 2013;3:2529.

16. Young RJ, Litière $S$, Lia $M$, et al. Predictive and prognostic factors associated with soft tissue sarcoma response to chemotherapy: a subgroup analysis of the European Organisation for Research and Treatment of Cancer 62012 study. Acta Oncol 2017;56:1013-20.

17. Kim JH, Cho SH, Kim EK, et al. Endobronchial malignant fibrous histiocytoma: case report of an unusual presentation and palliative flexible bronchoscopic resection. Respir Care 2013;58:e92-94.

18. Wang G, Sun M, Jiang Y, et al. Anlotinib, a novel small 
molecular tyrosine kinase inhibitor, suppresses growth and metastasis via dual blockade of VEGFR2 and MET in osteosarcoma. Int J Cancer 2019;145:979-93.

19. Bedi M, King DM, Charlson J, et al. Multimodality management of metastatic patients with soft tissue sarcomas may prolong survival. Am J Clin Oncol 2014;37:272-77.

20. Linde N, Casanova-Acebes M, Sosa MS, et al. Macrophages orchestrate breast cancer early dissemination and metastasis. Nat Commun 2018;9:21.

Cite this article as: $\mathrm{Xu} Z, \mathrm{Qu} W, \mathrm{Yu}$ Y. Primary giant cell malignant fibrous histiocytoma of the lung: a rare case report and literature review. Transl Cancer Res 2020;9(11):7350-7358. doi: $10.21037 /$ tcr-20-2297 